\title{
Demodulation Algorithm for Fiber Bragg Grating Sensors
}

\author{
Jianxin Zheng, ${ }^{1,2}$ Huadong Yang,,$^{1,2 *}$ Zichao Wang, ${ }^{1,2}$ and Zhiang Liu ${ }^{1,2}$ \\ ${ }^{1}$ Technology Center of CCCC Second Harbor Engineering Company Ltd., Wuhan 430040, China \\ ${ }^{2}$ CCCC Highway Bridge National Engineering Research Centre Co. Ltd, Beijing 100032, China
}

(Received November 24, 2021; accepted January 17, 2022)

Keywords: fiber Bragg grating, demodulation algorithm, variable step size, correlation coefficient

A demodulation algorithm is vital for a fiber Bragg grating (FBG) sensing system. In this paper, a novel demodulation algorithm based on the variable-step-size method and crosscorrelation algorithm is proposed to demodulate the wavelength of an FBG. By changing the step size of each calculation, the amount of calculation can be greatly reduced, and pm-level resolution can be achieved over the whole bandwidth. A simulation shows that the demodulation results are not affected by noise, and the algorithm can accurately demodulate the FBG wavelength in a large range. The stability of the algorithm is also tested using three FBG sensors, and the results show that the difference among 25 measurements is only $4 \mathrm{pm}$. Moreover, the results of temperature calibration experiments show that the sensitivity of the sensor is 41.9 $\mathrm{pm} /{ }^{\circ} \mathrm{C}$, which is similar to the value of $45 \mathrm{pm} /{ }^{\circ} \mathrm{C}$ provided by the manufacturer. The $R^{2}$ value of the fitting curve is 0.9994 , which means that the demodulation algorithm can be effectively used in sensing measurement.

\section{Introduction}

Fiber optic sensors have advantages including anti-electromagnetic interference, corrosion resistance, strong multiplexing ability, high sensitivity, portability, and flexibility, and have been used to monitor various physical parameters in complex and severe environments. ${ }^{(1-4)}$ A fiber Bragg grating (FBG) sensor, a type of wavelength-modulated fiber optic sensor, has unique advantages in addition to the above advantages. ${ }^{(5)}$ Owing to the wavelength modulation, changes in the light source intensity, link loss, and the electrical signal intensity of an optical fiber sensing system will not influence the measurement results. Therefore, FBG sensors are widely used to monitor physical parameters such as temperature, ${ }^{(6,7)}$ strain, ${ }^{(8)}$ and displacement in the aerospace, civil engineering, petroleum, and petrochemical fields. ${ }^{(9-11)}$

Accurate wavelength demodulation is the key to FBG sensing systems. At present, the conventional method is to scan the center wavelength of an FBG by adopting a narrow-linewidth tunable laser or a broadband light source combined with a tunable filter. ${ }^{(12-15)}$ The central wavelength of the tunable laser or the transmission wavelength of the tunable filter corresponding to the maximum detector signal intensity is considered as the central wavelength of the FBG. The above two methods have high requirements for the hardware such as the light source or

*Corresponding author: e-mail: 464179071@qq.com

https://doi.org/10.18494/SAM3741 
filter, and the wavelength resolution of the hardware is directly related to the accuracy of wavelength demodulation. At the same time, the influence of the external temperature on the light source or filter will also cause errors in demodulation results.

Therefore, the development of a wavelength demodulation algorithm with high demodulation accuracy and low requirements for the hardware and the external environment is of great significance for FBG sensing systems. In this paper, a wavelength demodulation algorithm based on variable-step-size cross-correlation is proposed through the study of the crosscorrelation algorithm. By constructing the FBG reflectance spectrum curve and carrying out the cross-correlation calculation with the real spectrum curve, the wavelength is obtained according to the correlation coefficient, and the variable-step-size algorithm is applied to reduce the amount of computation, thus realizing the rapid demodulation of the wavelength in a wide bandwidth range.

\section{Principles and Simulation Analysis}

\subsection{Principles}

The reflection spectrum curve of a virtual FBG should be constructed before measurement. In general, the reflection spectrum of an FBG can be approximated as a Gaussian curve expressed by the Gaussian function

$$
I=\alpha I_{0} \exp \left(-\frac{\left(\lambda-\lambda_{0}\right)^{2}}{2 B_{W}{ }^{2}}\right),
$$

where $\alpha$ is the reflectivity of the FBG, $I_{0}$ is the initial intensity of the incident light, $\lambda_{0}$ is the central wavelength of the reflection spectrum, and $B_{W}$ is the $3 \mathrm{~dB}$ width of the reflection spectrum.

Then, a virtual reflection spectrum sequence can be constructed as follows:

$$
X(n)=\sum_{i=1}^{n} \exp \left(-\frac{\left(\lambda_{i}-\lambda^{\prime}\right)^{2}}{2 B_{W}^{2}}\right)
$$

where $\lambda^{\prime}$ is the FBG center wavelength of the virtual reflection spectrum.

The actual reflection spectrum sequence $I(n)$ obtained by a photodetector can be expressed as

$$
I(n)=\alpha I_{0} \sum_{i=1}^{n} \exp \left(-\frac{\left(\lambda_{i}-\lambda_{0}\right)^{2}}{2 B_{W}{ }^{2}}\right),
$$

where $\lambda_{0}$ is the central wavelength of the FBG to be measured.

Then, the correlation coefficient $C$ between the actual reflection spectrum sequence and the virtual reflection spectrum sequence can be obtained as 


$$
C=\frac{\operatorname{cov}(X(n), I(n))}{\sigma_{X(n)} \sigma_{I(n)}},
$$

where $\sigma_{X(n)}$ is the standard deviation of $X(n)$ and $\sigma_{I(n)}$ is the standard deviation of $I(n)$.

When the center wavelength of the virtual reflection spectrum $\lambda^{\prime}$ is equal to the actual center wavelength $\lambda_{0}, \mathrm{C}$ takes its maximum value $C_{\max }$, and the value of $\lambda^{\prime}$ corresponding to $C_{\max }$ is the center wavelength $\lambda_{0}$.

\subsection{Principle of measurement}

According to the above measurement principles, the selected scanning step size will directly determine the accuracy of demodulation. The smaller the step size, the more accurate the demodulation result will be, but the amount of calculation will also increase rapidly. To obtain a demodulation accuracy of $1 \mathrm{pm}$ within a bandwidth range of $40 \mathrm{~nm}, 40000$ correlation calculations will be required, which is unsuitable for real-time demodulation. Therefore, in this paper, we propose a variable-step-size cross-correlation algorithm to achieve fast and highprecision wavelength demodulation, which can ensure demodulation accuracy and greatly reduce the amount of computation.

To illustrate the implementation process of the demodulation algorithm, we assume an FBG with a center wavelength of $1546.916 \mathrm{~nm}$ and attempt to approach a center wavelength within the bandwidth range of $1528-1568 \mathrm{~nm}$. The implementation steps are as follows:

Step 1: Firstly, to overcome the shortage of sampling points in a spectrometer or detector, a cubic spline interpolation algorithm is used for interpolation processing to make the curve smoother and improve the wavelength resolution. The normalized spectrum after treatment is shown in Fig. 1.

Step 2: The cross-correlation calculation is carried out as described above within the bandwidth range of $40 \mathrm{~nm}$ with a step size of $100 \mathrm{pm}$ to obtain the cross-correlation curve shown in Fig. 2 by fitting, and the wavelength value corresponding to the maximum correlation coefficient at this time is found to be $\lambda_{1}=1546.900 \mathrm{~nm}$.

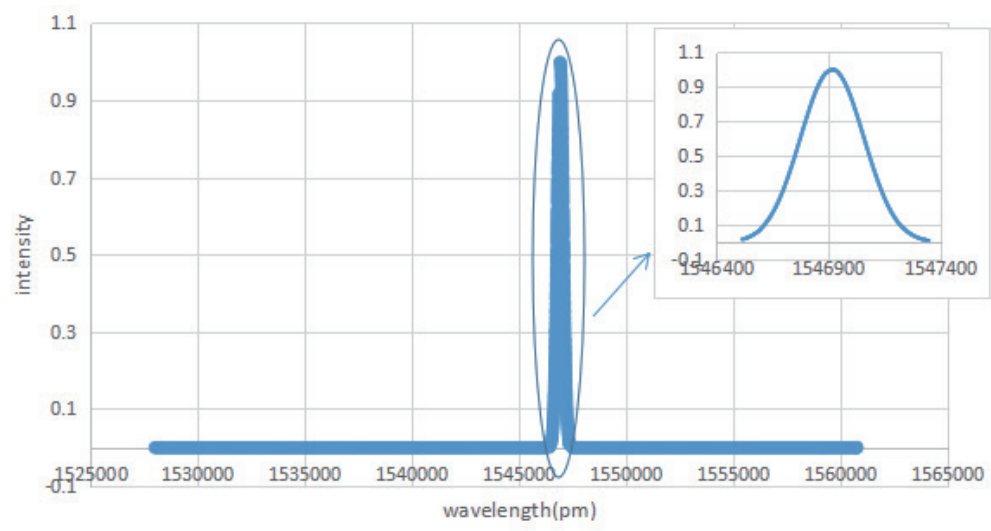

Fig. 1. (Color online) Normalized spectrum after processing. 


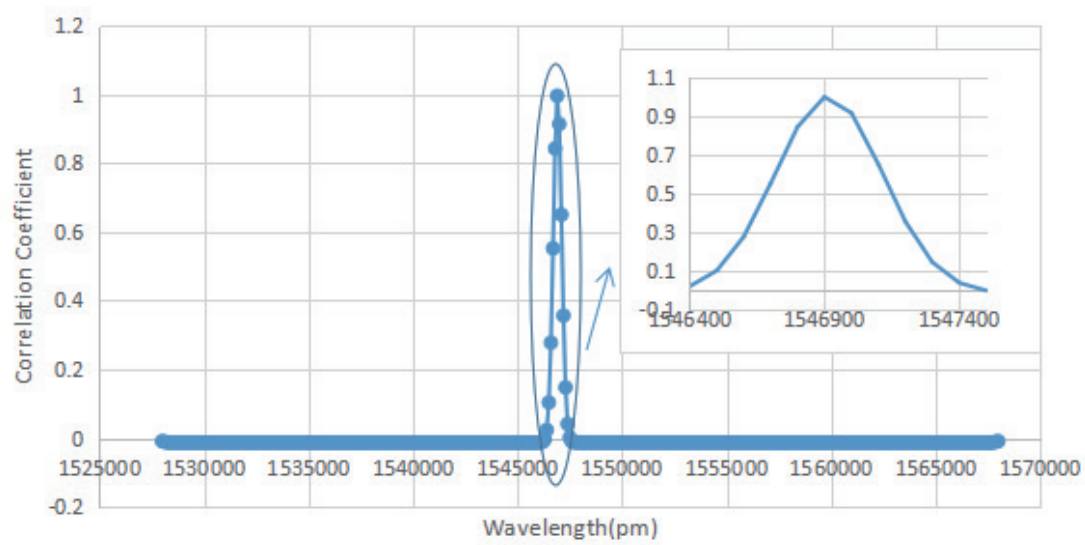

Fig. 2. (Color online) Correlation coefficient curve with step size of $100 \mathrm{pm}$.

Step 3: Considering that the above step size is $100 \mathrm{pm}$, the secondary search range is set from $(1546.900-0.2) \mathrm{nm}$ to $(1546.900+0.2) \mathrm{nm}$, and then the cross-correlation calculation is carried out with a step size of $1 \mathrm{pm}$. The obtained cross-correlation curve is shown in Fig. 3, and the wavelength corresponding to the maximum correlation coefficient at this time, $\lambda_{2}=1546.916$ $\mathrm{nm}$, is the wavelength to be demodulated.

After 800 cross-correlation calculations as described above, a demodulation accuracy of 1 $\mathrm{pm}$ is obtained within the bandwidth range of $40 \mathrm{~nm}$, and the amount of calculation is reduced by two orders of magnitude. The time required for the new demodulation method is only $0.8 \mathrm{~s}$, compared with $26.6 \mathrm{~s}$ for the traditional method. In addition, by increasing the number of variable-step-size operations, the amount of computation can be further reduced and the demodulation accuracy can be improved.

\subsection{Simulation analysis}

Each algorithm has a certain demodulation range. To ascertain the application range of this algorithm, continuous wavelength demodulation is performed in the entire wavelength range from 1528 to $1568 \mathrm{~nm}$. To more closely model an actual demodulation scene, Gaussian white noise is added to the simulation of a real signal spectrum as shown in Fig. 4.

Then the algorithm is applied to a demodulation experiment. The wavelength of the FBG is increased from 1528 to $1568 \mathrm{~nm}$ with a step size of $40 \mathrm{pm}$, and then the algorithm is applied to wavelength demodulation. By comparing the wavelength obtained by demodulation with the actual wavelength, the fitting curve can be obtained, and the demodulation error of the algorithm is also calculated.

It can be seen from Fig. 5(a) that the slope of the fitting curve is 1, which means that each demodulation value is exactly the same as the actual value at each time. Thus, the method can accurately demodulate the wavelength of the FBG from 1528 to $1568 \mathrm{~nm}$. In Fig. 5(b), we can see that the maximum simulation error is $1 \mathrm{pm}$ from 1528 to $1568 \mathrm{~nm}$. Therefore, the demodulation algorithm is applicable to FBGs of any wavelength. 


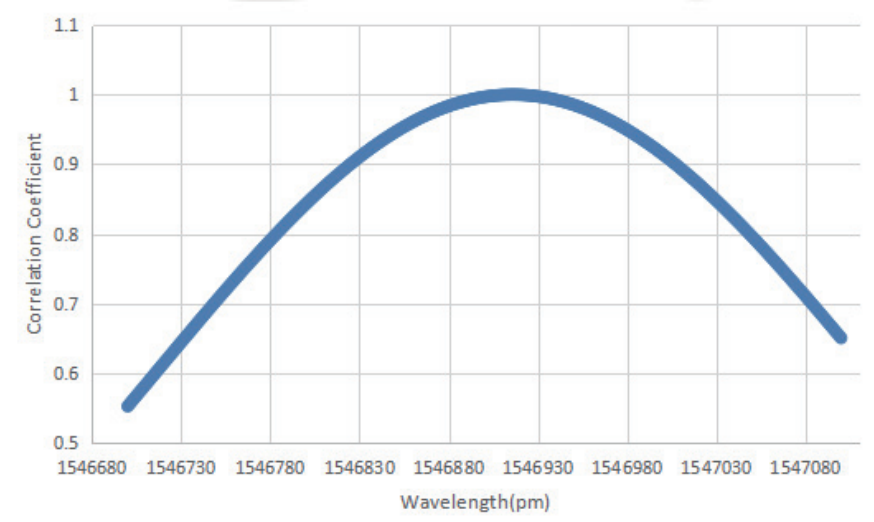

Fig. 3. (Color online) Correlation coefficient curve with step size of $1 \mathrm{pm}$.

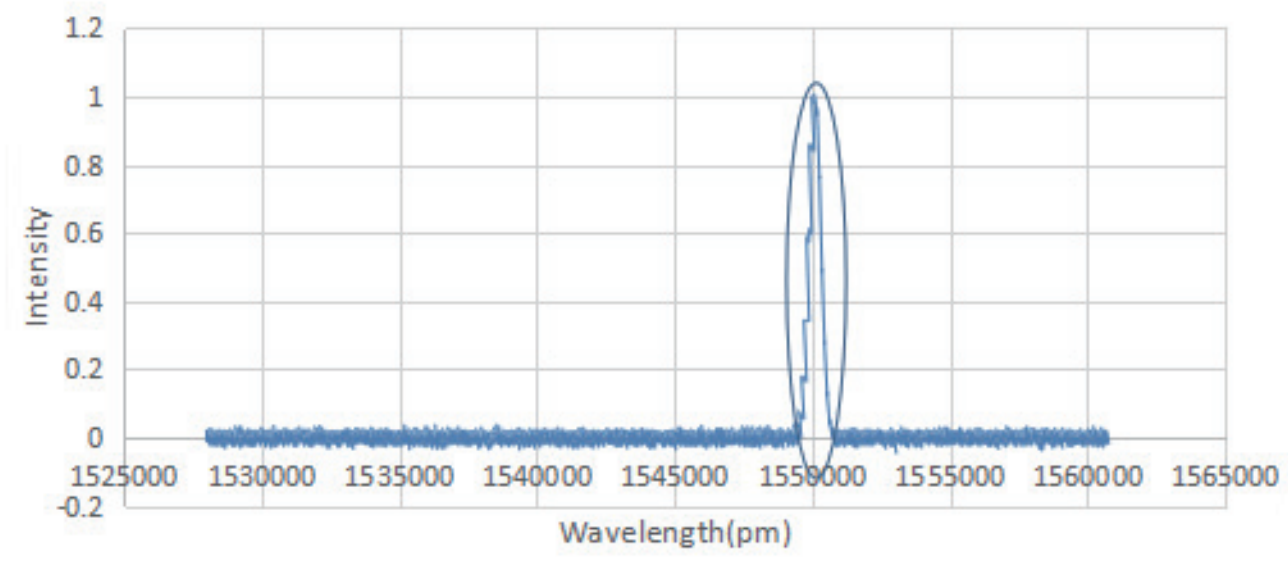

Fig. 4. (Color online) Signal spectrum of FBG with central wavelength of $1550.1 \mathrm{~nm}$.

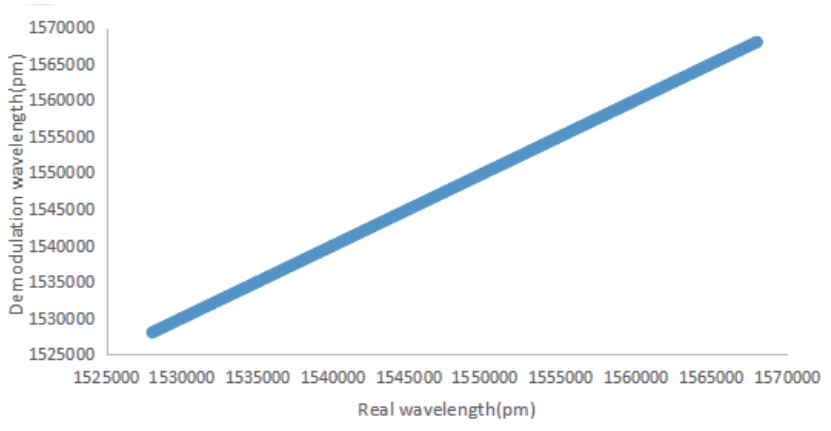

(a)

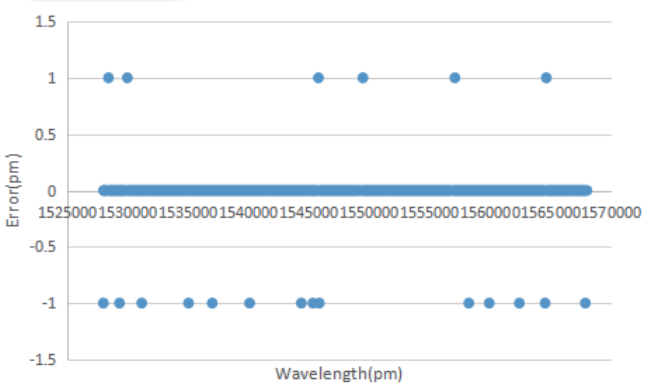

(b)

Fig. 5. (Color online) Simulation of demodulation: (a) relation between demodulation wavelength and actual wavelength; (b) relation between demodulation error and wavelength.

\section{Experiments and Results}

To verify the practical performance of the algorithm, three FBG sensors are used to carry out experiments. The sensing system is composed of a light source, FBG sensors, and signal 
acquisition and processing modules as shown in Fig. 6 . The reflectivity of the sensors is $0.05 \%$. The bandwidth of the light source is $40 \mathrm{~nm}(1528$ to $1568 \mathrm{~nm})$, the resolution of the spectrometer is $0.02 \mathrm{~nm}$, and both the light source and the spectrometer are packaged in an instrument case as shown in Fig. 6.

The spectra of the three sensors can be obtained using this experimental setup. However, owing to the resolution of the spectrometer, the curves are insufficiently smooth, and it is difficult to obtain pm resolution by direct demodulation. Therefore, before demodulation, cubic spline interpolation is used to process each spectrum. The processed spectra are shown in Fig. 7. The central wavelengths of the spectra of FBG \#1, FBG \#2, and FBG \#3 are about 1539.36, 1553.83 , and $1540.68 \mathrm{~nm}$, respectively, and the $3 \mathrm{~dB}$ bandwidth of the reflection spectra is about
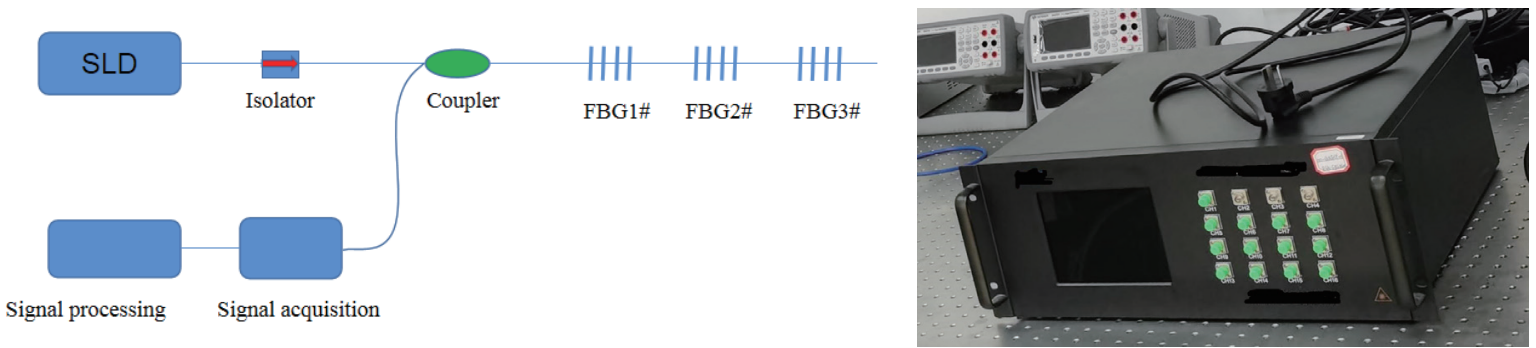

Fig. 6. (Color online) Experimental setup.

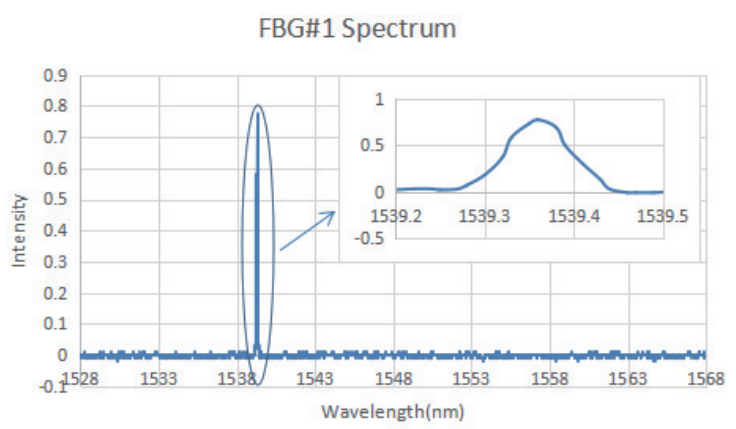

(a)

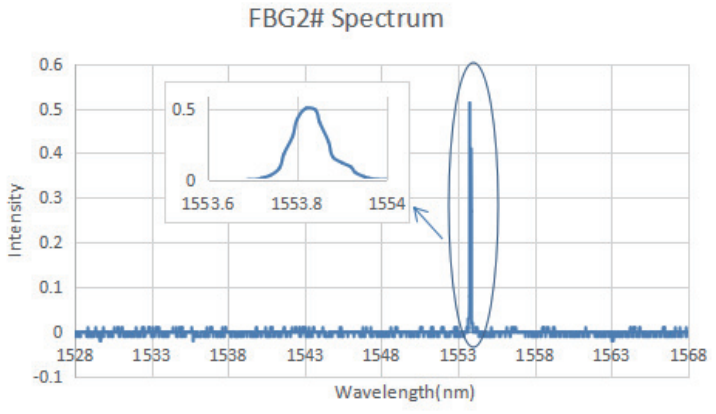

(b)

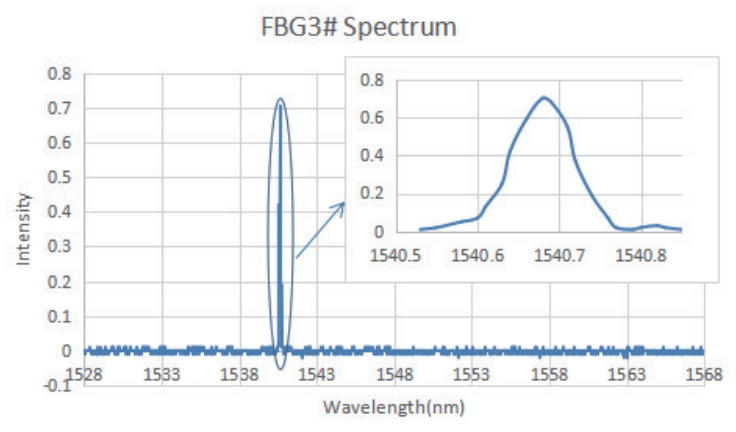

(c)

Fig. 7. (Color online) Reflection spectrum of FBGs: (a) FBG \#1, (b) FBG \#2, and (c) FBG \#3. 
$0.1 \mathrm{~nm}$.

To test the stability and resolution of the algorithm, continuous measurements are carried out in the same environment using the above experimental setup. The spectrometer continuously collects spectral data 25 times. Then, the spectral data in the wavelength range from 1528 to $1568 \mathrm{~nm}$ are demodulated by the algorithm and accurate wavelength values are obtained. The results are shown in Fig. 8.

It can be seen from Fig. 8 that the central wavelengths of the three sensors are demodulated correctly and have good stability. Among the 25 measurements, the difference between the maximum and minimum measured values is only $4 \mathrm{pm}$. According to our analysis, the error may originate from the fluctuation of the ambient temperature and the fact that the reflection spectra in Fig. 7 are not ideal Gaussian curves.

Next, temperature calibration experiments are carried out. A weak FBG (wFBG) with a reflectivity of $0.05 \%$ fabricated from TPEE material is used. The temperature coefficient is about $45 \mathrm{pm} /{ }^{\circ} \mathrm{C}$, which includes $10.8 \mathrm{pm} /{ }^{\circ} \mathrm{C}$ attributed to the thermo-optical effect of the optical fiber and about $35 \mathrm{pm} /{ }^{\circ} \mathrm{C}$ attributed to the thermal expansion of the TPEE (Hatcui) material. The reflection spectrum of the wFBG is shown in Fig. 9.

The temperature is varied from 40 to $44{ }^{\circ} \mathrm{C}$ in steps of $1{ }^{\circ} \mathrm{C}$ with each temperature maintained for $30 \mathrm{~min}$, and the spectrum is measured three times at each temperature. The measurement results are shown in Table 1 . Then the average values from Table 1 are used to calibrate the

FBG1\#

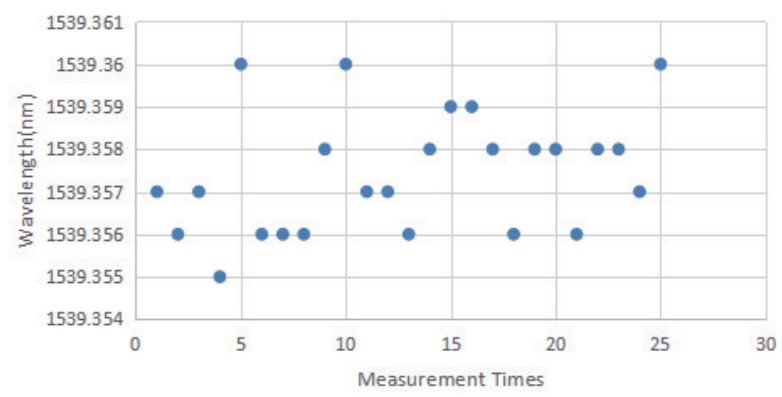

(a)

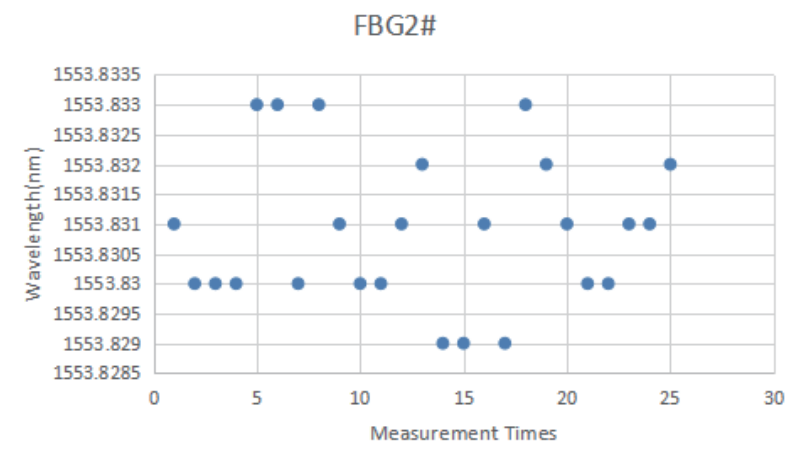

(b)

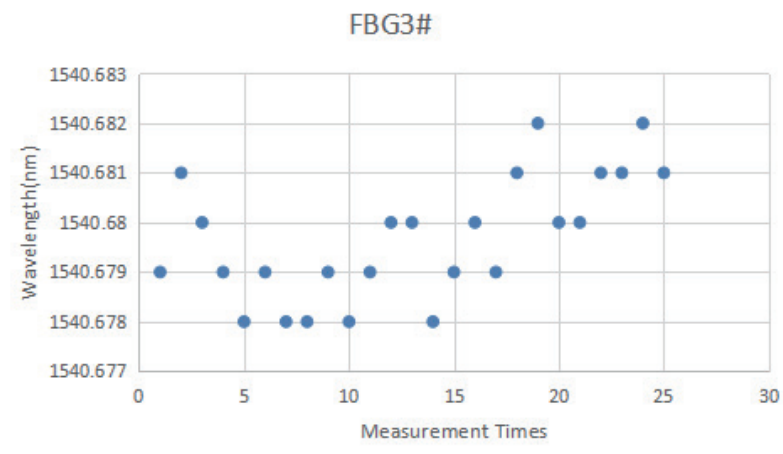

(c)

Fig. 8. (Color online) Measurement results of FBGs: (a) FBG \#1, (b) FBG \#2, and (c) FBG \#3. 


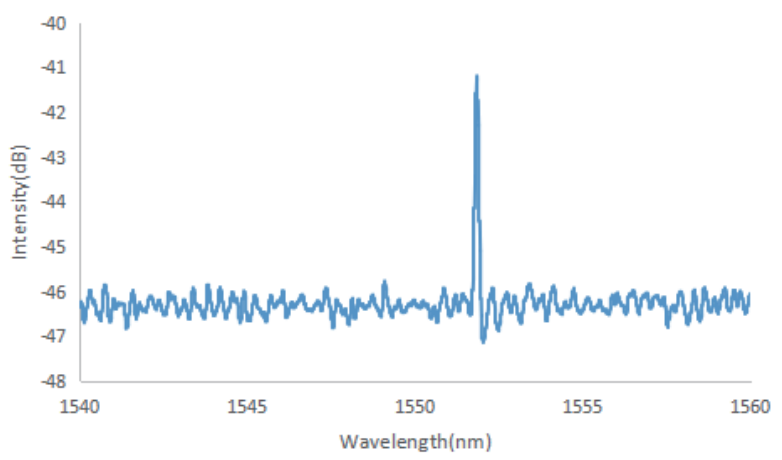

Fig. 9. (Color online) Reflection spectrum of the wFBG.

Table 1

Measurement results of temperature calibration experiments.

\begin{tabular}{lcccc}
\hline Temperature $\left({ }^{\circ} \mathrm{C}\right)$ & \multicolumn{3}{c}{ Central wavelength $(\mathrm{pm})$} & Average value $(\mathrm{pm})$ \\
\hline 40 & 1552664 & 1552661 & 1552667 & 1552664 \\
41 & 1552709 & 1552706 & 1552714 & 1552710 \\
42 & 1552748 & 1552750 & 1552755 & 1552751 \\
43 & 1552786 & 1552795 & 1552791 & 1552791 \\
44 & 1552834 & 1552829 & 1552837 & 1552833 \\
\hline
\end{tabular}

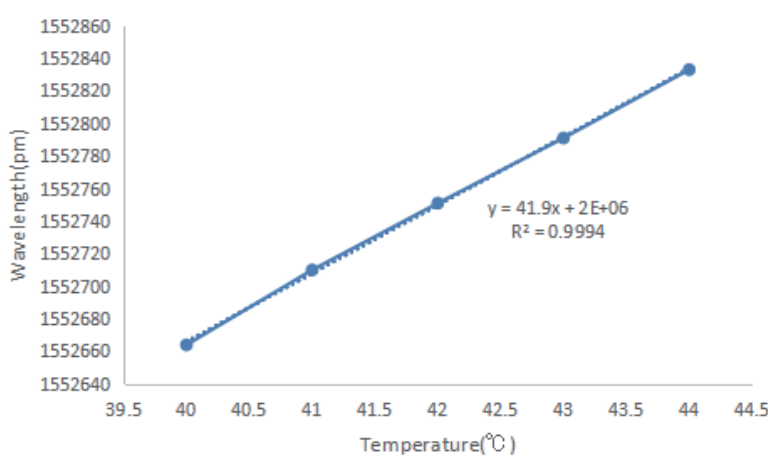

Fig. 10. (Color online) Change in central wavelength during heating.

relationship between the temperature and the central wavelength, as shown in Fig. 10.

The $R^{2}$ value of the calibration fitting curve is 0.9994 , indicating high linearity of the fitting result. The sensitivity of the sensor is $41.9 \mathrm{pm} /{ }^{\circ} \mathrm{C}$, which is similar to the value of $45 \mathrm{pm} /{ }^{\circ} \mathrm{C}$ provided by the manufacturer. Thus, the demodulation algorithm can be effectively used in sensing measurement.

\section{Conclusions}

A novel demodulation algorithm based on the variable-step-size cross-correlation algorithm is proposed to demodulate the FBG wavelength. By changing the step size while performing a 
series of cross-correlation calculations, pm-level resolution in a large bandwidth range can be achieved after a relatively small number of cross-correlation calculations. The simulation results show that the demodulation results are not affected by the broadening of the FBG reflection spectrum, and the algorithm can accurately demodulate FBG wavelengths in a large range. The results of stability tests demonstrate that the algorithm has good stability and high-precision demodulation with only $4 \mathrm{pm}$ fluctuation during 25 measurements. The $R^{2}$ value of the calibration fitting curve is 0.9994 and the sensitivity of the sensor is $41.9 \mathrm{pm} /{ }^{\circ} \mathrm{C}$ in temperature calibration experiments, similar to the provided value of $45 \mathrm{pm} /{ }^{\circ} \mathrm{C}$. This means that the demodulation algorithm can be effectively used in sensing measurements. As a result, the novel algorithm can achieve pm-level resolution, high measurement accuracy, and a large dynamic demodulation range, giving it excellent application prospects.

\section{Acknowledgments}

This work was supported by the Major Technology Innovation Project of Hubei Province (No. 2018AAA031).

\section{References}

1 V. Soto and M. López-Amo: J. Phys.: Photonics 1 (2019) 042002. http://doi.org/10.1088/2515-7647/ab3f0e

2 K. Grattan and T. Sun: Sens. Actuators, A 82 (2000) 40. http://doi.org/10.1016/S0924-4247(99)00368-4

3 C. Maya and K. Vinod: Opt. Fiber Technol. 64 (2021) 102580. http://doi.org/10.1016/J.YOFTE.2021.102580

4 H. Yang, X. Tong, C. Zhang, Q. Guo, and P. Hu: Appl. Opt. 56 (2017) 7450. http://doi.org/10.1364/AO.56.007450

5 C. Li, J. Tang, C. Cheng, L. Cai, and M. Yang: Photonic Sens. 11 (2021) 91. http://doi.org/10.1007/S13320-021$\underline{0615-8}$

6 R. Yan, G. Sang, B. Yin, S. Wu, M. Wang, B. Hou, M. Gao, and R. Chen: Opt. Express 20 (2021) 13530. http:// doi.org/10.1364/OE.421782

7 H. Fu, S. Wang, H. Chang, and Y. You: Opt. Fiber Technol. 60 (2020) 102369. http://doi.org/10.1016/j. yofte.2020.102369

8 J. Yang, P. Hou, C. Yang, and N. Yang: Sensors 21 (2021) 3492. http://doi.org/10.3390/S21103492.

9 Y. Zhan, F. Lin, Z. Song, Z. Sun, and M. Yu: Optik 229 (2020) 166122. http://doi.org/10.1016/j.ijleo.2020.166122

10 A. Samavati, M. Velashjerdi, A. F.Ismail M.H.D. Othman, G. Eisaabadi B., A. Awang, Z. Samavati, A. Rostami, and N. Yahyad: Sens. Actuators, A 318 (2020) 112428. http://doi.org/10.1016/j.sna.2020.112428

11 L. Zeng, X. Sun, Z. Chang, Y. Hu, and J. Duan: Chin. Opt. Lett. 19 (2020) 030602. https://www.osapublishing. org/col/abstract.cfm?URI= col-19-3-030602

12 J. Wang, G. Song, X. Liu, C. Wang, and T. Liu: Procedia Eng. 26 (2011) 765. http://doi.org/10.1016/j. proeng.2011.11.2235

13 L. Nunes,B. Olivieri,C. Kato, L. Valente, and A. Braga: Sens. Actuators, A 138 (2007) 341. https://doi. org/10.1016/j.sna.2007.05.009

14 P. Dai, Y. Zhou, L. Wang, S. Liu, X. Zhang, and X. Chen: Chin. Opt. Lett. 18 (2020) 071201. https://doi. org $/ 10.3788 /$ col202018.071201

15 Y. Guo, C. Yu, Y. Ni, and H. Wu: Opt. Fiber Technol. 54 (2020) 102129. https://doi.org/10.1016/j. yofte.2019.102129 
